\section{Quand les cellules cancéreuses sont accros à un gène normal}

Laurence Lamy
Metabolism Branch,

National Cancer Institute,

National Institutes of Health, NIH,

9000 Rockville Pike, Bethesda,

Maryland 20892, États-Unis.

lamyl@mail.nih.gov

Le myélome multiple, un phénotype unique mais des altérations génétiques multiples Le myélome multiple reste une maladie incurable malgré les récents progrès de la chimiothérapie et I'utilisation des transplantations de cellules souches hématopoïétiques. Cette hémopathie représente environ $1 \%$ de tous les cancers, et la survie moyenne est de trois ans à dater du diagnostic. C'est un cancer qui touche les

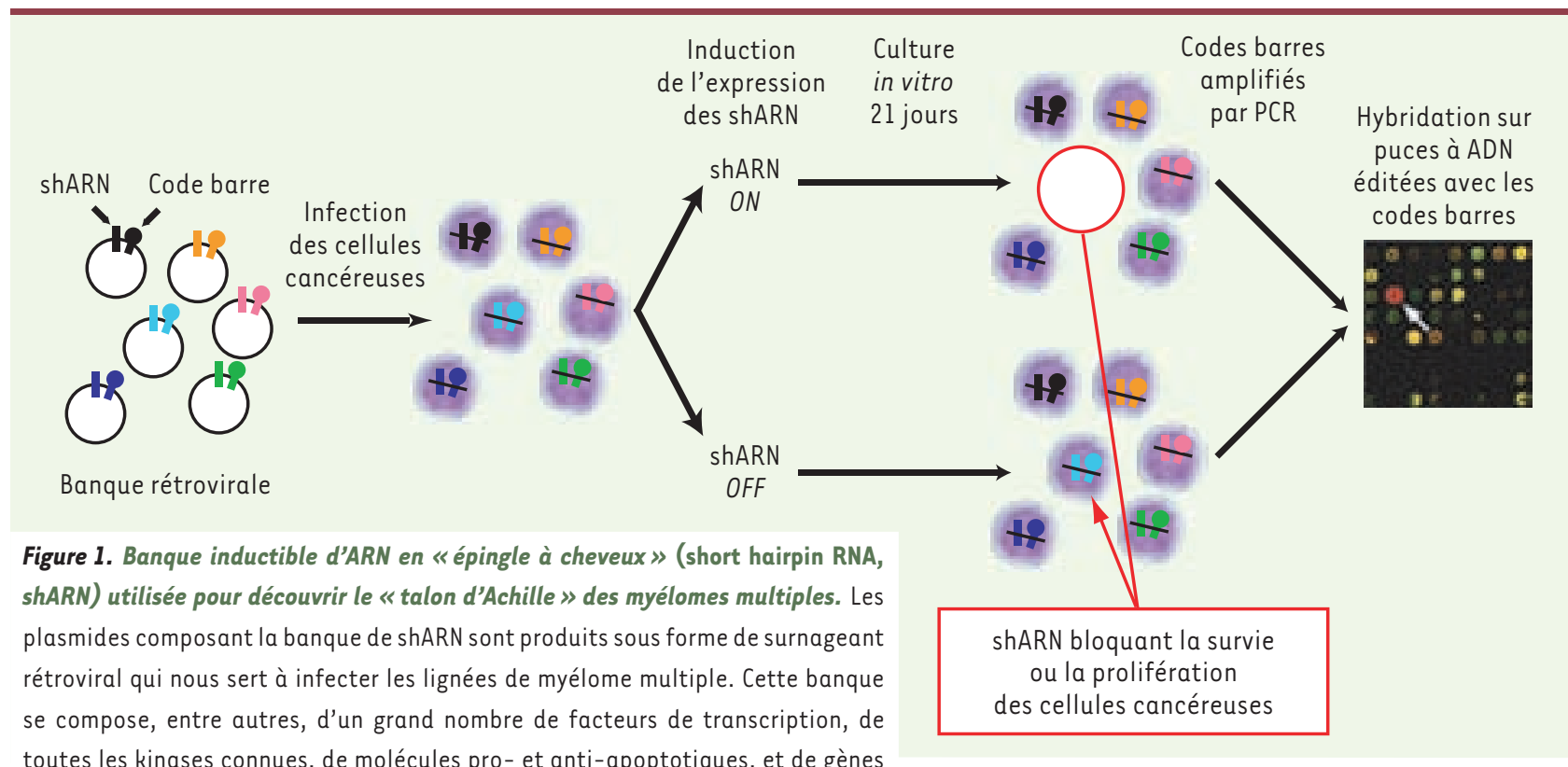
toutes les kinases connues, de molécules pro- et anti-apoptotiques, et de gènes dont l'expression varie selon le type de lymphome mais dont la fonction reste à définir. Nous avons utilisé un système inductible car les shARN qui inhibent l'expression de tels gènes peuvent s'avérer toxiques pour la cellule. En principe, chaque cellule n'est infectée que par un seul rétrovirus, ce qui implique qu'un gène unique, différent pour chaque cellule, est éteint lors de l'induction des shARN. À chaque shARN est associé un « code barre » de 60 nucléotides qui lui est spécifique. Ce code barre permet par la suite de retrouver les clones qui ont proliféré ou au contraire ont disparu par rapport à une culture témoin. Après avoir sélectionné les cellules qui expriment la banque rétrovirale, les cellules sont divisées en deux sous-populations, l'une dont l'expression des shARN est induite, et l'autre, non induite, qui sert de contrôle négatif. Les cellules qui expriment un shARN inhibant l'expression d'un gène indispensable à leur prolifération ou à leur survie disparaissent de la population de cellules infectées. Après 21 jours de traitement, l'ADN génomique des cellules induites et non induites est purifié, et la séquence du code barre est amplifiée par PCR. Les produits de PCR des cultures induites et non induites sont marqués à l'aide de fluorochromes différents et co-hybridés sur des puces à ADN éditées avec les séquences de 60 nucléotides des codes barres. L'intensité du signal fluorescent reflète alors l'abondance de chaque shARN dans les cultures induites et non induites et désigne les gènes dont l'expression est indispensable à la prolifération et à la survie des myélomes. 
cellules plasmatiques (ou plasmocytes), qui sont des lymphocytes $B$ entrés dans leur dernière phase de différenciation à la suite d'une infection, et dont le rôle est de produire les immunoglobulines spécifiques des antigènes rencontrés. Les plasmocytes normaux et tumoraux sont localisés dans la moelle osseuse où ils trouvent un microenvironnement favorable à leur survie [3] $(\rightarrow)$.

$(\rightarrow)$ Voir l'article de Bertrand Huard, page 5 de ce numéro

Lors d'un myélome, le plasmocyte transformé prolifère de façon incontrôlée et envahit la moelle osseuse d'os distants; les plasmocytes malins continuent de se multiplier, remplaçant progressivement la moelle osseuse saine. Cette prolifération s'accompagne de la surproduction d'immunoglobulines monoclonales intactes (IgG, IgA, IgD, ou $\lg \varepsilon$ ) ou de protéines de Bence-Jones (chaînes légères et monoclonales libres) responsables d'une hyperviscosité du sang, d'une insuffisance rénale et d'un déficit immunitaire dû à un appauvrissement de la moelle osseuse. Les plasmocytes tumoraux sont également responsables de la sécrétion de cytokines qui activent les ostéoclastes, entraînant la destruction du tissu osseux et une hypercalcémie.

Le développement de traitements basés sur le ciblage d'oncogènes spécifiquement dérégulés dans ces cancers s'est heurté à la grande hétérogénéité de la maladie. En effet, les myélomes multiples sont classés en 7 catégories, selon que les plasmocytes présentent une hyperdiploïdie ou, ce qui est le cas pour près de la moitié de ces hémopathies, la translocation d'oncogènes [cyclines D1 et D3, cMaf, MafB (musculoaponeurotic fibrosarcoma oncogene homolog B) et MMSET/FGFR3, fibroblast growth factor receptor 3] au niveau du promoteur de la chaîne lourde des immunoglobulines [4].

\section{L'addiction des cellules plasmatiques cancéreuses à IRF4, un gène normal}

Nous avons mis au point très récemment un criblage génétique basé sur l'utilisation de l'ARN interférence afin de trouver les gènes nécessaires à la survie ou à la prolifération des cellules cancéreuses (Figure 1) [5]. Cette approche a été appliquée à des lignées de myélomes multiples caractérisées par des altérations

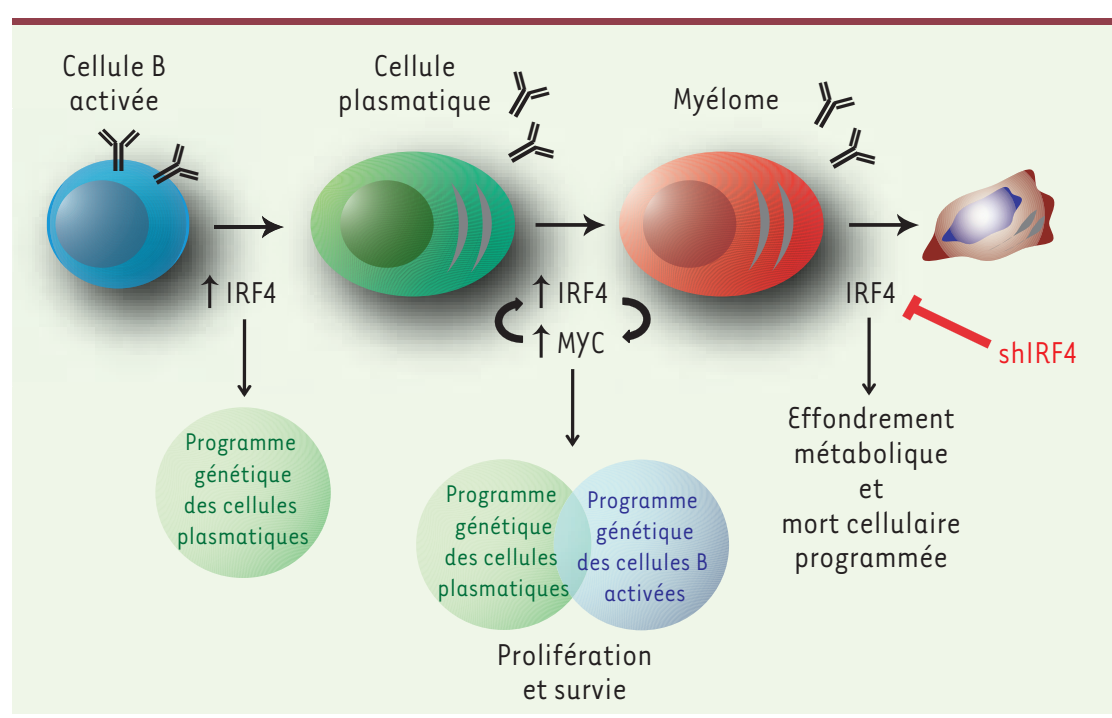

Figure 2. Implication d'IRF4 dans la dérégulation du répertoire génétique des cellules plasmatiques. Dans les myélomes multiples, IRF4 est engagé dans une boucle de régulation positive avec $M Y C$, ce qui conduit à la fusion du répertoire génétique des cellules plasmatiques et des cellules $B$ activées. Inhiber l'expression ou la fonction d'IRF4 revient à inhiber l'expression aberrante de ces gènes qui sont devenus indispensables à la survie et à la prolifération de ces cellules et représente un traitement potentiel des myélomes multiples. génétiques différentes. Or, quel que soit le type de myélome multiple, nous avons trouvé que l'inhibition d'IRF4 (interferon regulatory factor 4 ) est létale pour les cellules de myélome, mais sans effet dans la majorité des lymphomes testés [6].

IRF4 est un facteur de transcription exprimé essentiellement dans les cellules du système immunitaire, en particulier dans les lymphocytes et les cellules dendritiques [7, 8]. La lignée lymphocytaire B exprime IRF4 de façon biphasique: il est exprimé dans les cellules $B$ immatures de la moelle osseuse, réprimé dans les centroblastes des centres germinatifs des organes lymphoïdes secondaires, puis ré-exprimé au cours de la maturation des cellules $B$ en cellules plasmatiques dont dérivent les myélomes multiples [9]. Dans les cellules plasmatiques, IRF4 et d'autres facteurs de transcription tels que Blimp-1 (B lymphocyte-induced maturation protein 1 ) et XBP-1 (X-box binding protein 1 ) entraînent l'arrêt du cycle cellulaire et favorisent la synthèse et la sécrétion des immunoglobulines.

Étonnamment, le gène IRF4 n'est ni muté, ni transloqué, ni amplifié dans la majorité des cellules de myélomes testées, ce qui implique que le produit du gène normal est nécessaire à la survie de ces cellules cancéreuses. Cependant, IRF4 n'induit pas le même profil d'activation dans les cellules plasmatiques normales et dans les plasmocytes de myélomes. L'étude montre que près de $25 \%$ des gènes activés par IRF4 dans les myélomes multiples correspondent à des gènes surexprimés dans les cellules $B$ activées, alors que ces gènes sont normalement réprimés dans les cellules plasmatiques, qui ne prolifèrent pas. L'un de ces gènes est particulièrement important, puisqu'il s'agit du facteur de transcription MYC, un coordinateur clé du métabolisme et de la croissance cellulaire. Dans les myélomes multiples, MYC serait lui-même responsable de l'induction aberrante d'une partie de ces gènes impliqués dans la glycolyse, la respiration mitochondriale, la progression du cycle cellulaire et la régulation de la sénescence cellulaire. Une autre observation importante est qu'IRF4 lui- 
même est une cible de MYC, cette boucle de régulation positive étant responsable d'un emballement du système où chacun de ces deux gènes renforce l'expression de l'autre. II est donc probable que l'inhibition d'IRF4 dans les myélomes provoque leur mort en induisant un effondrement du métabolisme de la cellule (Figure 2).

Mais comment expliquer qu'IRF4, qui n'est pas un oncogène, ait accès à un répertoire génétique élargi dans les myélomes? On peut envisager différentes hypothèses. D'une part, le locus MYC est fréquemment amplifié ou transloqué au voisinage du locus des immunoglobulines dans les myélomes primaires et les lignées [10]. Cet événement pourrait être le point de départ de la surexpression d'IRF4 dans les myélomes et l'activation oncogénique de MYC permettrait à IRF4 de reproduire le profil d'activation caractéristique observé dans les cellules B. D’autre part, des altérations épigénétiques dans les myélomes pourraient permettre à IRF4 d'accéder à des locus normalement silencieux dans les cellules plasmatiques.

\section{Cibler les gènes normaux}

constituant le talon d'Achille

des cellules cancéreuses,

une myriade de nouveaux traitements?

Les myélomes multiples étant caractérisés par une grande hétérogénéité, leur traitement ne peut que cibler des fonctions très générales [11]. Par exemple, l'inhibiteur du protéasome bortezomib (Velcade ${ }^{\circledR}$ ) empêche la dégradation des protéines ubiquitinylées et aboutit à l'augmentation du stress cellulaire avec, à terme, l'inhibition du facteur de transcription NF- $\kappa B$ et l'activation des caspases, entraînant l'apoptose des cellules. La thalidomide et ses analogues sont des immunomodulateurs qui inhibent la production de TNF- $\alpha$ (tumor necrosis factor), des interleukines- $1 \beta,-6$, -12 et stimulent l'activité cytotoxique des cellules natural killer (NK) et des cellules T à l'encontre des cellules de myélome. Ces molécules seraient aussi capables d'inhiber l'adhérence des cellules de myélome à leur microenvironnement, de réduire la sécrétion de facteurs de croissance et de survie par les cellules stromales et d'exercer une activité anti-angiogénique. Mais ces traitements, même s'ils permettent d'allonger la durée de vie du patient, ne sont pas curatifs et s'accompagnent d'effets secondaires. Notre étude met en évidence la dépendance du processus tumoral vis-à-vis d'un facteur de transcription normal, exprimé aussi bien dans les cellules tumorales que dans les cellules plasmatiques dont elles dérivent, et de ce fait commun à tous les types de myélomes. Cette découverte apporte une contrepartie à l'hypothèse de «l'addiction des cellules cancéreuses aux oncogènes », qui suggère que certains cancers dépendent de l'activité d'un gène muté pour survivre ou proliférer [12]. Les travaux de séquençage des génomes de cellules tumorales permettent d'identifier ces oncogènes et leur inactivation suffirait, en théorie, à détruire la tumeur. En revanche, le gène codant IRF4 n'est pas muté dans les myélomes, mais les cellules sont tout de même dépendantes de sa capacité à activer de façon inappropriée des voies de signalisation normalement muettes. La recherche classique de mutations, translocations ou amplifications ne permet pas de détecter de tels gènes, mais la généralisation des criblages génétiques à grande échelle devrait permettre d'allonger la liste de ces «non-oncogènes » indispensables à la survie des cellules cancéreuses et qui pourraient constituer de nouvelles cibles thérapeutiques plus efficaces et plus spécifiques. $\diamond$

When cancer cells are addicted to a regular gene

\section{RÉFÉRENCES}

1. Laget S, Defossez PA. Le double jeu de l'épigénétique: cible et acteur du cancer. Med Sci (Paris) 2008 ; $24: 725-30$.

2. Bertucci F, Finetti P, Cervera N, Birnbaum D. Classification pronostiquedu cancer du sein et profils d'expression génique sur puces à ADN. Med Sci (Paris) 2008 ; 24 : 599-606.

3. Burjanadze M, Roosnek E, Passweg J, Huard J. APRIL fait le printemps des plasmocytes. Med Sci (Paris) $2008 ; 24: 5-8$.

4. Zhan F, Huang $\mathrm{Y}$, Colla $\mathrm{S}$, et al. The molecular classification of multiple myeloma. Blood 2006; 108: 2020-8.

5. Ngo VN, Davis RE, Lamy L, et al. A loss-of-function RNA interference screen for molecular targets in cancer. Nature 2006 ; 441 : 106-10.

6. Shaffer AL, Emre NC, Lamy L, et al. IRF4 addiction in multiple myeloma. Nature 2008 ; 454 : 226-31.

7. Lehtonen A, Veckman V, Nikula T, et al. Differential expression of IFN regulatory factor 4 gene in human monocyte-derived dendritic cells and macrophages. J Immunol 2005 ; 175 : 6570-9.

8. Mittrucker HW, Matsuyama T, Grossman A, et al. Requirement for the transcription factor LSIRF/IRF4 for mature B and T lymphocyte function. Science 1997 ; $275: 540-3$.

9. Falini B, Fizzotti M, Pucciarini A, et al. A monoclonal antibody (MUMlp) detects expression of the MUMI/ IRF4 protein in a subset of germinal center B cells, plasma cells, and activated T cells. Blood 2000 ; 95 : 2084-92.

10. Shou Y, Martelli ML, Gabrea A, et al. Diverse karyotypic abnormalities of the c-myc locus associated with cmyc dysregulation and tumor progression in multiple myeloma. Proc Natl Acad Sci USA $2000 ; 97$ : 228-33.

11. Merchionne F, Perosa F, Dammacco F. New therapies in multiple myeloma. Clin Exp Med 2007 ; 7 : 83-97.

12. Weinstein IB, Joe A. Oncogene addiction. Cancer Res $2008 ; 68: 3077-80$.

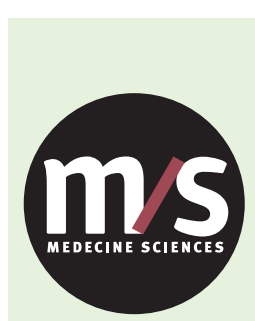

Tarifs d'abonnement M/S - 2009

Abonnez-vous

à Médecine/Sciences
$>$ Grâce à $m / s$, vous vivez en direct les progrès des sciences biologiques et médicales

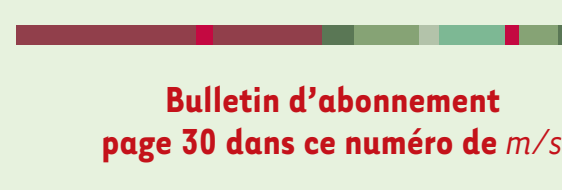

.

